\title{
The common hyperlipidaemias
}

\author{
BARRY LEWIS* \\ Ph.D., M.D., F.R.C.P., M.R.C.Path. \\ Department of Chemical Pathology, Royal Postgraduate Medical School, \\ Hammersmith Hospital, London W12 OHS
}

\begin{abstract}
Summary
The treatment of primary hyperlipidaemic states is reviewed. While several metabolically distinct forms of hyperlipidaemia exist, it is possible to classify patients into three groups for the purpose of therapy.

Group 1 is defined as hypercholesterolaemia (due to raised levels of $\beta$-lipoprotein). In Group 2 the level of endogenous triglyceride is increased, with or without hypercholesterolaemia. In the rare Group 3, there is exogenous hypertriglyceridaemia. A satisfactory response can be attained in at least $90 \%$ of cases, using dietary and drug treatment.
\end{abstract}

\section{Introduction}

Evidence has been presented that hyperlipidaemia is one of the major sources of risk of ischaemic heart disease. Preventive measures directed against this risk factor fall into two categories. One is the public health approach, which seeks to shift the frequency distribution of serum lipid levels in the population towards the left, decreasing the proportion of the community whose lipid concentrations lie in the high-risk part of the distribution. The only feasible means of achieving this is dietary, and involves us in providing the public, the food manufacturer and the government with realistic advice on desirable nutritional patterns.

The second category affects us in our directly clinical role and involves the detection of hyperlipidaemia in our patients and their relatives, with a view to optimal treatment. Therapy has to be appropriate to the particular metabolic disorder present: no single measure is effective against all hyperlipidaemic states. As hyperlipidaemia (however defined) is among the commonest of metabolic disorders, we are concerned with potentially large numbers of patients. The clinical and laboratory investigations should therefore be as simple as possible.

\section{Recognition of hyperlipidaemia}

Only a minority of hyperlipidaemic patients present with specific diagnostic features such as xanthomas, but it adds only a moment to routine

* Present address: Department of Chemical Pathology, St Thomas's Hospital Medical School, London, S.E. 1. examination to feel for tendon xanthomas on the dorsa of the hands and in the Achilles tendons, and to look for cutaneous xanthomas on the knees, elbows and buttocks, and in the palmar creases. An uncommon but characteristic sign is retinal lipaemia. Less exotic signs are corneal arcus and xanthelasmas, which are suggestive of hyperlipidaemia when they occur in young individuals (before age 35) but can occur with apparently normal lipid levels. Severe hypertriglyceridaemia can lead to attacks of acute pancreatitis, and recurrent abdominal pain may be the presenting symptom of this group of metabolic disorders. Gall-stones also occur with abnormal frequency in this group of patients. Abdominal 'angina' is an occasional manifestation.

Hyperlipidaemia may present with rheumatological features. An acute migratory large-joint polyarthritis occurs in patients with severe hypercholesterolaemia, resembling rheumatic fever; the presence of tendon xanthomas, and the family history, suggest the correct diagnosis. Hyperuricaemia is frequently associated with the common endogenous form of hypertriglyceridaemia and overt gouty arthritis is sometimes seen.

All too often, hyperlipidaemia is first recognized in the course of investigating patients who already have manifest ischaemic heart disease or peripheral vascular disease. This could be regarded as an advanced stage in the natural history of this group of metabolic defects; but there is justification for detecting and treating hyperlipidaemia even at this juncture, for hypercholesterolaemia remains a risk factor for recurrence of new ischaemic events in patients who have experienced myocardial infarction (Coronary Drug Project Research Group, 1974). Surgical treatment of coronary artery atherosclerosis is now making it possible to relieve myocardial ischaemia in selected patients. It seems appropriate to deal with correctable risk factors in patients so treated.

Another cardiovascular manifestation of severe hypercholesterolaemia is aortic stenosis. Xanthomatous involvement of the valve, or gross atherosclerosis of the ascending aorta can produce valvar 
or supravalvar stenosis with a pressure gradient; coronary disease may or may not also be present.

Of patients with ischaemic heart disease, $56 \%$ were found to have hyperlipoproteinaemia (defined as levels exceeding the 90th percentile) in a recent study (Lewis et al., 1974a). Similar findings have been obtained in peripheral vascular disease (Lewis et al., 1974b). Most types of hyperlipoproteinaemia were over-represented in these series compared with apparently-healthy controls. In young patients, up to $80 \%$ may be so affected. In these studies, raised levels of very low density lipoprotein (VLDL, pre- $\beta$ lipoprotein) were the commonest finding, followed by increased concentrations of low density lipoprotein (LDL, $\beta$-lipoprotein), and by raised levels of both VLDL and LDL. LDL is the major cholesterolbearing lipoprotein of plasma, while the VLDL carries most of the circulating endogenous triglyceride and also a moderate proportion of the plasma cholesterol.

The forms of hyperlipidaemia accompanying ischaemic heart disease have recently been the subject of important genetic studies (Goldstein et al., 1973; Nikkilä and Aro, 1973; Patterson and Slack, 1972). All have agreed that the commonest heritable disorder of lipid metabolism in such patients is an indeterminate one in which members of affected families may show raised levels of either VLDL or LDL or of both lipoproteins, 'multiple-type' hyperlipoproteinaemia or familial combined hyperlipidaemia. In terms of serum lipids, increased concentrations of both cholesterol and triglyceride are commoner than elevation of either lipid alone.

\section{Screening for hyperlipidaemia}

Many hyperlipidaemic individuals are detected as a result of screening procedures, either in the course of 'health-screening' or of 'profiling' of patients. An important aspect of the investigation of hyperlipidaemic patients is the measurement of serum lipid levels in their accessible first-degree relatives. The early diagnosis of hyperlipidaemia may be so achieved.

Whole-population screening for heart disease risk factors has its advocates. Further evidence is desirable as to the benefits of this approach, and at the practical level, facilities do not exist at present for the management of large numbers of hyperlipidaemic individuals. But it is possible to define population groups in whom risk factor screening on a relatively modest scale is possible. Amongst these are patients developing cardiac or peripheral ischaemia, especially before the age of 60 , and their first-degree relatives. Patients prone to secondary hyperlipidaemia include those with diabetes, gout and chronic renal failure. There is evidence that the frequency of ischaemic heart disease is grossly increased in such conditions, and correction of associated hyperlipidaemia and hypertension is indicated. Evidence is presented elsewhere in this symposium that the presence of multiple risk factors leads to a striking increase in proneness to ischaemic heart disease. A convincing case can therefore be made for assessing serum lipid levels in patients with hypertension.

The common hyperlipidaemias (Fredrickson and Levy, 1972; Beaumont et al., 1970; Lewis, 1976).

Definition of the upper limit of normality is necessary for clinical purposes, but is a somewhat arbitrary matter. At the author's lipid clinic the cutoff points are the 90th percentiles for apparently healthy adults aged $20-39$ years. For cholesterol, the level is $250 \mathrm{mg} / 100 \mathrm{ml}(6.5 \mathrm{mmol} / 1)$ in both sexes. The upper limit for triglyceride in men is $2 \cdot 1 \mathrm{mmol} / 1$ $(185 \mathrm{mg} / 100 \mathrm{ml})$ and in women $1.5 \mathrm{mmol} / 1(135$ $\mathrm{mg} / 100 \mathrm{ml}$ ). Blood samples are drawn without venous stasis, with the subject sitting quietly, having fasted for 12-14 hr. For baseline measurements, the patient should be on his normal diet for at least 2 weeks, and should not have taken lipid-active drugs for a month. Lipid studies should be deferred for 3 months after major illness.

The initial classification distinguishes primary and secondary hyperlipidaemias. Clinical examination and simple laboratory investigations usually suffices. for this purpose. Common underlying causes ar hypothyroidism, diabetes, alcoholism, cholestasis, uraemia, nephrotic syndrome and gout. No fundamental classification of primary hyperlipoprotein aemias is yet possible. In order to provide optimal therapy of primary hyperlipidaemic states, these must be classified into a minimum of three categories; the first two, and possibly all of these are genetically and metabolically heterogeneous.

\section{(1) The hyper- $\beta$-lipoproteinaemia group (type IIa)}

This is characterized by hypercholesterolaemia with normal triglyceride levels, and is a common disorder. In most patients hyper- $\beta$-lipoproteinaemia appears to be the result of the interaction between multiple genes and environmental factors, of which diet is likely to be important ('polygenic type') Much more rarely it is due to a single dominant gene (monogenic hyper- $\beta$-lipoproteinaemia). Both forms are undoubted risk factors for ischaemic heart disease. At least $50 \%$ of men with the latter disorder develop ischaemic heart disease by the age of 50 years (Slack, 1969), although it appears somewhat less hazardous in women. The concentration of $\beta$-lipoprotein in the arterial intima is increased several fold, paralleling plasma levels (Onitiri et al., 1976). Ischaemic heart disease presents at a significantly younger age in patients with this disorder 
than in those with normal serum lipoproteins (Lewis et al., 1974a) and ischaemic ECG changes are common even in asymptomatic individuals (Carlson, Ekelund and Olsson, 1975).

In many respects these forms of hyper- $\beta$-lipoproteinaemia differ. Physical signs in the polygenic variety are limited to xanthelasmas and corneal arcus, while patients with the monogenic form commonly develop tendon xanthomas. In the homozygous state, and in severely affected heterozygotes, the latter form is also characterized by cutaneous xanthomas (flattened, planar lesions on extensor surfaces) and by aortic stenosis.

The diagnosis of hyper- $\beta$-lipoproteinaemia is probable when 'pure' hypercholesterolaemia is accompanied by any of the above clinical features. Occasionally, moderately raised cholesterol levels are due to hyper- $\alpha$-lipoproteinaemia; this too may be familial, but is unassociated with abnormal physical signs. It is not a source of vascular risk, and it has even been suggested that it is protective against ischaemic heart disease. When in doubt, the presence of raised levels of $\beta$-lipoprotein should be confirmed by quantitative measurement; if this is not available lipid measurements and qualitative lipoprotein electrophoresis are performed.

In the monogenic disorder, $\beta$-lipoprotein levels are raised from birth, although total serum cholesterol concentration is not always elevated, since a reciprocal disease in $\beta$-lipoprotein level is often marked in neonates. In adults, this disorder leads to serum cholesterol concentrations of 8-15 mmol/1 (310 $580 \mathrm{mg} / 100 \mathrm{ml}$ ) in the heterozygous state, and 14-28 $\mathrm{mmol} / 1(540-1080 \mathrm{mg} / 100 \mathrm{ml})$ in the homozygote. The polygenic form of hyper- $\beta$-lipoproteinaemia is usually but not always milder, cholesterol levels most often ranging from 6.5 to $9 \mathrm{mmol} / 1$ (250-350 $\mathrm{mg} / 100 \mathrm{ml}$ ).

Pathogenesis of the polygenic form is unclear. In the monogenic disorder, there is some evidence that impaired catabolism of $\beta$-lipoproteins and also enhanced synthesis occur. Various defects in cellular uptake of this lipoprotein have been described in studies on skin fibroblasts; these may lead to a decreased rate of breakdown of $\beta$-lipoprotein, and to escape of cholesterol synthesis from its normal repression by the lipoprotein.

\section{(2) The endogenous hypertriglyceridaemia group}

Hypertriglyceridaemia is the characteristic feature of this prevalent group of disorders, while cholesterol levels may be normal or increased. Lipoprotein studies are essential to distinguish this group from exogenous hypertriglyceridaemia.

Endogenous hypertriglyceridaemia due to raised levels of pre- $\beta$-lipoprotein (VLDL) is a common finding in patients with ischaemic heart disease, alone or with hypercholesterolaemia. While most prospective surveys have indicated that raised triglyceride levels are predictive of ischaemic events, there is disagreement as to whether this is an independent effect or whether it results from the weak but significant positive correlation between triglyceride and cholesterol concentrations. From other sources of evidence however, it appears likely that elevated concentrations of VLDL do predispose to cardiac and peripheral ischaemia. Asymptomatic men with high levels of VLDL, like those with elevation of LDL concentrations or of both VLDL and LDL, show ischaemic post-exercise ECG changes far more often than do those with mid-range levels of these lipoproteins (Carlson et al., 1975). The mean age at which symptoms of cardiac or peripheral ischaemia first present is earlier in patients with raised VLDL levels than in those without lipoprotein abnormalities (Lewis et al., 1974a). There is evidence too, that VLDL, or its metabolic products, enters the arterial intima during life, and that its concentration there is positively correlated with its plasma levels. Although VLDL is the major transport protein for endogenous triglyceride, it also has a substantial cholesterol component, which may be significant in relation to atherogenesis.

Patients with endogenous hypertriglyceridaemia occasionally develop eruptive xanthomas and retinal lipaemia. Commoner associated features are obesity, mild glucose intolerance, hyperinsulinism and hyperuricaemia; but none of these is invariably present.

When hypertriglyceridaemia is accompanied by normal or modestly-increased cholesterol levels, the underlying lipoprotein abnormality is likely to be an increase in VLDL levels (type IV). This is the commonest form of endogenous hypertriglyceridaemia. In patients with severe hypertriglyceridaemia, elevated VLDL concentration is sometimes associated with chylomicronaemia (type V). Other variants of endogenous hypertriglyceridaemia are those in which both triglyceride and cholesterol levels are substantially increased. This is most often due to elevation of both VLDL and LDL concentration (type IIb); less often, there is an accumulation in plasma of partly delipidated 'remnants' of VLDL and chylomicra (type III). In this disorder, it is usual to find rounded tuberous xanthomas over the elbows, and orange-coloured planar xanthomas in the palmar creases.

In the diagnosis of these disorders, it is necessary to characterize the lipoprotein pattern underlying the hyperlipidaemia; this is principally to exclude the rare group $\mathbf{3}$ disorder in which chylomicronaemia is the typical finding. The simplest laboratory method for making this distinction is the 'stored serum test'. After hypertriglyceridaemic serum has stood for 18 hr at $4^{\circ} \mathrm{C}$, it may show a variety of appearances. 
Chylomicra float to the surface, producing a creamy upper layer with clear subnatant: this is the characteristic appearance of exogenous hypertriglyceridaemia (group 3). The particles containing endogenous triglyceride do not float under these circumstances, hence the sample remains diffusely lactescent (turbid), although sometimes with an additional creamy upper layer. Lipoprotein electrophoresis will also permit recognition of the various forms of hypertriglyceridaemia.

In some patients, endogenous hypertriglyceridaemia appears to be due to overproduction of VLDL, while others show defective removal of triglyceride from plasma. Hyperinsulinism is not a constant feature of the disorder but it is widely believed that at least in a subgroup of patients with endogenous hypertriglyceridaemia, excessive secretion of VLDL by the liver is caused by the insulin excess. There is little evidence that dietary carbohydrate plays a role in the pathogenesis of this group of disorders.

\section{(3) Exogenous hypertriglyceridaemia}

Severe hypertriglyceridaemia and relatively moderate hypercholesterolaemia are the usual findings in this condition, the excessive amounts of plasma lipids being transported entirely or chiefly in chylomicra (type I hyperlipoproteinaemia). As mentioned earlier, this is most readily diagnosed by the appearance of stored serum: a floating creamy layer with clear subnatant is characteristic. The electrophoretic pattern of serum lipoproteins is often diagnostic.

Patients usually present in infancy or childhood but the diagnosis may not be made until adult life. Attacks of abdominal pain are the commonest presenting feature, the other common manifestation being eruptive xanthomas.

The condition is due to recessively-inherited deficiency of the enzyme lipoprotein lipase, present chiefly in muscle and adipose tissue; this enzyme hydrolyses circulating triglyceride permitting its uptake from plasma. Dietary fat therefore accumulates in plasma, and lipaemia is much diminished within 3-5 days of instituting a fat-free diet. Lipoprotein lipase may be assayed in plasma after injection of heparin, which releases it into the circulation.

Despite the severity of the hyperlipidaemia in this disorder, there is no evidence that it predisposes to premature vascular disease. The purpose of treatment is to avert pancreatitis and xanthomatosis.

Treatment of hyperlipidaemias (Tabaqchali et al., 1974; Lewis, 1976).

The majority of patients respond readily to relatively simple measures, involving dietary treatment alone or with a few well tried drugs. Correction of any treatable cause for secondary hyperlipidaemia will usually ameliorate or correct the associated lipid abnormality, the commonest examples being diabetes, myxoedema and alcoholism. However the hyperlipidaemia of gout does not as a rule decrease when hyperuricaemia is treated. Another general measure of major importance is substantial weight reduction in obese patients; this often suffices to control endogenous hypertriglyceridaemia and is of benefit in most forms of combined hyperlipidaemia. Responsiveness to lipid-lowering drug therapy is sometimes markedly improved by dealing effectively with obesity. Although hard data are lacking, it seems prudent to replace oestrogen-containing oral contraceptive combinations by other means of birth control in hyperlipidaemic patients.

Hyper- $\beta$-lipoproteinaemia of polygenic type is usually responsive to a fat-modified diet. This is designed to provide normal calorie requirements, a reduced total fat intake of $35-37 \%$. Cholesterol intake is limited to $250-300 \mathrm{mg} / \mathrm{day}$. The main feature of this diet is limitation of saturated fat intake and its partial replacement by polyunsaturated fats, so that the ratio of polyunsaturated to saturated fatty acids is about $\mathbf{1 . 8}$. This is achieved by limiting the intakes of meat fat, dairy fat and eggs, and by increasing consumption of seed oils, suitable margarines and fish. This diet reduces serum choles 8 terol levels by $15-25 \%$ and often decreases tri glyceride levels substantially. For patients who dळे not respond adequately to diet, clofibrate, $1 \mathrm{~g}$ twice daily, is usually effective.

The rarer monogenic form of hyper- $\beta$-lipoproteinaemia is less readily corrected. In addition to the fat-modified diet, treatment with one or more drugs is usually required. Cholestyramine is at present the drug of choice, taken with meals in a daily dose of 16-28 g/day. It may be necessary to add clofibrate, or nicotinic acid which appears more effective but also more troublesome. To minimize flushing and dyspepsia with nicotinic acid, dosage is increased over a period of weeks from $0.25 \mathrm{~g} /$ day to 4-6 g/day in divided doses. In the extremely rare patient with cholesterol levels exceeding about $14 \mathrm{mmol} / 1$ and with cutaneous and tendon xanthomas, therapy poses great difficulty. Ischaemic heart disease often presents in childhood. Most of the patients are homozygous for monogenic hyper- $\beta$-lipoproteinaemia. Some respond quite well to diet, cholestyramine and nicotinic acid. In others, surgical ileal by-pass appears effective. Experimentally, portacaval shunt has been successful, as has frequent plasma exchange. In a further subgroup, probably metabolically distinct, there is a remarkably good response to diet alone.

Endogenous hypertriglyceridaemia in obese patients responds well to adequate weight reduction, but there is a tendency for total cholesterol levels (or LDL-cholesterol concentration) to rise as triglyceride 
falls. In the initially-lean patient, or after weight reduction, it is worth introducing an isocaloric fatmodified diet; reduction of triglyceride and cholesterol levels is often considerable. If drug treatment proves necessary, clofibrate is usually effective although nicotinic acid appears to have a greater effect in some patients.

The treatment of exogenous hypertriglyceridaemia is by a diet low in ordinary fats. Adults most often tolerate 20-25 g fat/day. The diet can be made more palatable by addition of medium chain triglyceride, which is not transported in chylomicra.

\section{References}

Bequmont, J.L., Carlson, L.A., Cooper, G.R., Fejfar, Z., Fredrickson, D.S. \& STrasser, T. (1970) Classification of hyperlipidemias and hyperlipoproteinemias. Bulletin of the World Health Organization, 43, 891.

Carlson, L.A., Ekelund, L.G. \& Olsson, A.G. (1975) Frequency of ischaemic exercise ECG changes in symptomfree men with various forms of primary hyperlipaemia. Lancet, ii, 1 .

Coronary Drug Project Research Group (1974) Factors influencing long-term prognosis after recovery from myocardial infarction-three year findings of the Coronary Drug Project. Journal of Chronic Diseases, 27, 267.

FredRICKSON, D.S. \& LEVY, R.I. (1972) Familial hyperlipoproteinemia. In: The Metabolic Basis of Inherited Disease. 3rd Edn (Ed. by J. B. Stanbury, J. B. Wyngaarden and D. S. Fredrickson), pp. 545-614. New York, McGraw Hill.
Goldstein, J.L., Schrott, H.G., Hazzard, W.R., Bierman, E.L. \& Motulsky, A.G. (1973) Hyperlipidemia in coronary heart disease. II. Genetic analysis of lipid levels in 176 families and delineation of a new inherited disorder, combined hyperlipidemia. Journal of Clinical Investigation, 52, 1544.

LEwIS, B. (1976) The Hyperlipidaemias: Clinical and Laboratory Practice. Oxford: Blackwell.

lewis, B., Chait, A., Oakley, C.M., Krikler, D., Carlson, L.A., Ericsson, M., Boberg, J., Mancini, M., Oriente, P., Paggi, E., Micheli, H., Malczewski, B., Weisswange, A. \& Pometta, D. (1974b) Plasma lipoprotein abnormalities in normal and atherosclerotic subjects in four cities. In: Atherosclerosis III. (Ed. by G. Schettler and A. Weizel), p. 839. Berlin: Springer.

Lewis, B., Chait, A., OAKley, C.M.O., Wootton, I.D.P., Krikler, D.M., Onitiri, A., Sigurdsson, G. \& February, A. (1974a) Serum lipoprotein abnormalities in patients with ischaemic heart disease: comparisons with a control population. British Medical Journal, 3, 489.

Nikkilä, E.A. \& Aro, A. (1973) Family study of serum lipids and lipoproteins in coronary heart disease. Lancet, i, 954.

Onitiri, A.C., Lewis, B., Bentall, H., Jamieson, C., WISHEART, J. \& FARIS, I. (1976) Lipoprotein concentrations in serum and in biopsy samples of arterial intima: a quantitative comparison. Atherosclerosis, 23, 513.

Patterson, D. \& Slack, J. (1972) Lipid abnormalities in male and female survivors of myocardial infarction and their first degree relatives. Lancet, i, 393.

SLACK, J. (1969) Risks of ischaemic heart-disease in familial hyperlipoproteinaemic states. Lancet, ii, 1380.

Tabaqchali, S., Chait, A., Harrison, R. \& Lewis, B. (1974) Experience with simplified scheme of treatment of hyperlipidaemia. British Medical Journal, 1, 377. 